\title{
Uplink Admission Control in WCDMA Based on Relative Load Estimates
}

\author{
Fredrik Gunnarsson, Erik Geijer Lundin \\ Gunnar Bark, Niclas Wiberg, Ericsson Radio Systems AB \\ Control \& Communication \\ Department of Electrical Engineering \\ Linköpings universitet, SE-581 83 Linköping, Sweden \\ WWW: http://www.control.isy.liu.se \\ E-mail: fred@isy.liu.se, geijer@isy.liu.se
}

22nd February 2002

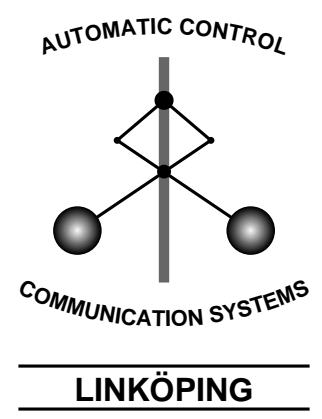

Report no.: LiTH-ISY-R-2414

Submitted to International Conference on Communications 2002

Technical reports from the Control \& Communication group in Linköping are available at http://www. control.isy.liu.se/publications. 


\begin{abstract}
When operating a cellular radio system at nearly full capac-ity, admitting yet another user may jeopardize the stability of the system as well as the performance of the individual users. Therefore, proper admission control is crucial. Prior art includes algorithms which are lim-iting the number of users or the uplink interference per cell. Both are known to yield roughly the same performance, but the former is difficult to configure, and the latter is based on a quantity, which is hard to mea-sure accurately. The core idea in this work is to predict the relative load of the system directly, given that a user is admitted. Then, the user will be admitted if the predicted load in the specific cell, and in its neighbors, is below some threshold. The proposed uplink relative load estimate is focused on WCDMA. It utilizes measurements readily available in that system, either periodically scheduled or from handover events. Multiservices are naturally handled, and availability of high data-rate services are automatically limited with respect to coverage, compared to services of lower data-rate. Simulations indicate that the admission control operates satisfactory in different traffic situations with a universal parameter setting. Furthermore, the reporting overhead with periodical measurements can be avoided, since handover event-driven measurements yield roughly the same performance.
\end{abstract}

Keywords: WCDMA, uplink load, estimation, handover measurements, noise rise 


\title{
Uplink Admission Control in WCDMA Based on Relative Load Estimates
}

\author{
Fredrik Gunnarsson $^{k, * *}$, Erik Geijer-Lundin', Gunnar Bark $^{k *}$, Niclas Wiberg $^{k *}$ \\ Ericsson Radio Systems AB and Linköpings universitet
}

\begin{abstract}
When operating a cellular radio system at nearly full capacity, admitting yet another user may jeopardize the stability of the system as well as the performance of the individual users. Therefore, proper admission control is crucial. Prior art includes algorithms which are limiting the number of users or the uplink interference per cell. Both are known to yield roughly the same performance, but the former is difficult to configure, and the latter is based on a quantity, which is hard to measure accurately. The core idea in this work is to predict the relative load of the system directly, given that a user is admitted. Then, the user will be admitted if the predicted load in the specific cell, and in its neighbors, is below some threshold. The proposed uplink relative load estimate is focused on WCDMA. It utilizes measurements readily available in that system, either periodically scheduled or from handover events. Multiservices are naturally handled, and availability of high data-rate services are automatically limited with respect to coverage, compared to services of lower data-rate. Simulations indicate that the admission control operates satisfactory in different traffic situations with a universal parameter setting. Furthermore, the reporting overhead with periodical measurements can be avoided, since handover event-driven measurements yield roughly the same performance.
\end{abstract}

\section{INTRODUCTION}

Admission and congestion control are important radio resource management mechanisms used to maintain acceptable service quality of existing connections. Admitting too many users results in a situation where the mutual interference between the connections degrade the quality of service. If such a situation arises, appropriate congestion control discontinues the service or reduces the data rate of some connections, to save the others. In general, it is more infringing to interrupt a service than to deny it in the first place.

The core idea of admission control is to admit users only if there are available resources to support their service requirements. A natural admission control strategy in a single service system (e.g., voice) is to limit the absolute number of users (or the number of links when users are in soft handover) in each cell, and only admit a new user if below this limit. This is referred to as hard capacity. The strategy can also be used in multi-service systems, by considering higher data-rate users as multiple low data-rate users capacity-wise. An alternative is to base the admission control on measurements or estimates (typically the total received power or interference) relating to the actual load of the system, which is referred to as soft capacity [1].

Many uplink soft capacity algorithms are based on total received power or the relation of the same to the thermal noise at the base stations. Such strategies include [1], [2], [3], [4], [5],

(*) are with the Division of Control and Communications, Dept. of EE, Linköpings universitet, SE-581 83 LINKÖPING, SWEDEN,

(**) are with Ericsson Radio Systems AB, Research and Innovation P.O. Box 1248, SE-581 12 LINKÖPING, SWEDEN.

Email: fredrik.gunnarsson@era.ericsson.se,geijer@isy.liu.se, gunnar.bark@era.ericsson.se,niclas.wiberg@era.ericsson.se
[6], [7], [8], [9]. An alternative is to use SIR measurements at the base station, which is further explored in [10], [11]. Admission control for multimedia traffic is the main issue in [12], [13], [14], [15]. Most of the aforementioned papers consider the situation in the specific cell only, while some also consider the situation in its neighbors. The load of the cellular radio system is truly a spatial quantity, and therefore hard to describe by a single number. However, some efforts are made in [16], [17], [18].

Hard capacity algorithms are easy to implement and therefore intuitive. Simulations in [3] show roughly the same performance as with soft capacity. However, it is difficult to determine the optimal hard capacity limits, which probably are different for different classes of cells, environments, equipment etc. Additionally, it should also be set conservatively to minimize the risk of overload in essentially all likely load situations. The critical problems with algorithms based on the total interference and noise powers are that it is difficult to separate the noise from the interference in measurements, and that the measurement accuracy is crude and time-varying. The latter is mainly due to component and temperature variations.

In this contribution, we discuss uplink admission control based on a measure of the relative (or fractional) load [1], [2], [17] of the system and estimates thereof using only available measurements. Hence, the focus is on radio aspects and the aim is to avoid too high an uplink interference relative the noise. A user is only admitted if the predicted loads of the cell and its neighbors are all below a threshold. Essentially we consider mobile-assisted measurements, either periodically scheduled or from handover events. A similar strategy, but for uplink packet access control, is considered in [19]. The idea is further explored here for real-time, connectionoriented services, and extended to also include soft handover. The results are also reported in [20]. Section III presents relevant models and notation. These are utilized in Section III. where we are more elaborate about capacity and relative load of cellular radio systems. These quantities are typically neither fully observable, nor directly controllable in practical implementations. Some tractable admission control algorithms based on relative load estimates are also discussed. The estimates and corresponding admission algorithms are evaluated in simulations with a hard capacity algorithm as a reference in Section IV and Section V provides some conclusive remarks.

\section{System Model}

Most physical quantities in this paper can be expressed using either logarithmic (e.g., $\mathrm{dB}$ or $\mathrm{dBm}$ ) or linear scale. To avoid confusion we will employ the convention of indicating linearly scaled values with a bar. Thus $\bar{g}_{i j}$ is a value in linear 
scale and $g_{i j}$ the corresponding value in logarithmic scale.

Consider an uplink situation in a cellular radio system, with $B$ base stations. (In this work, we will denote different sectors at the same cell site as different base stations.) Assume that the $M$ active mobile stations are transmitting using the powers $\bar{p}_{i}(t), i=1, \ldots, M$. The signal power from mobile station $i$ and base station $j(j=1, \ldots, B)$ is attenuated by the power gain $\bar{g}_{i j}(t)(<1)$. The uplink propagation situation in a cellular network can thus be described by the power gains, which together form the uplink gain matrix

$$
\overline{\boldsymbol{G}}(t)=\left[\bar{g}_{i j}(t)\right]=\left(\begin{array}{ccc}
\bar{g}_{11}(t) & \cdots & \bar{g}_{1 B}(t) \\
\vdots & \ddots & \vdots \\
\bar{g}_{M 1}(t) & \cdots & \bar{g}_{M B}(t)
\end{array}\right)
$$

This matrix is most likely not square, since there are more mobile stations than base stations in a cellular radio system. If the mobile station $i$ is connected to base station $j_{i}$, this base station will experience a carrier power $\bar{C}_{i}(t)=\bar{p}_{i}(t) \bar{g}_{i j_{i}}(t)$. All the received signals and thermal noise (with power $\bar{v}_{j}(t)$ ) at the base station combine to the total interference power (total received power):

$$
\bar{I}_{j}^{\mathrm{tot}}(t)=\bar{v}_{j}(t)+\sum_{i=1}^{M} \bar{g}_{i j}(t) \bar{p}_{i}(t)
$$

The perceived quality is related to the carrier-to-interference ratio (CIR) defined by

$$
\bar{\gamma}_{i}(t)=\bar{C}_{i}(t) /\left(\bar{I}_{i}^{\mathrm{tot}}(t)-\bar{C}_{i}(t)\right)
$$

For presentational ease, we will in some sections instead use the carrier-to-total-interference ratio (CTIR), $\bar{\beta}_{i}(t)=$ $\bar{C}_{i}(t) / \bar{I}_{i}^{\text {tot }}(t)$. In a simplistic situation the quality of a specific service, for example in terms of data rate or bit error rate, can be related to a required CIR, denoted target $C I R, \bar{\gamma}_{i}^{t}(t)$. This target is possibly reconsidered regularly by outer control loops [17]. The outer loop update rate is typically orders of magnitude faster than the admission control, and the changes are relatively small. Therefore, the target SIR:s will be considered constant.

An admitted mobile station is allocated

- a connection to a base station (typically, the one with most favorable propagation conditions, i.e., highest $\bar{g}_{i j}$ ).

- a waveform (in DS-CDMA, a code).

- a transmission power $\bar{p}_{i}(t)$. The aim is to update the power so that CIR is equal to target CIR, $\bar{\gamma}_{i}(t)=\bar{\gamma}_{i}^{t}$.

These allocations are regularly reconsidered by radio resource management algorithms. Power control is much faster than admission control. When studying admission control, it is therefore natural to assume perfect power control, i.e., $\bar{\gamma}_{i}(t)=$ $\bar{\gamma}_{i}^{t}$.

DS-CDMA allows a mobile station to be connected to a multitude of base stations at different cell sites (soft handover) or to several base stations at the same cell site (softer handover). Denote the set of connected base stations of mobile station $i$ by $K_{i}$. When in softer handover, the cell site can combine the received signals in an optimal manner. Using this maximum ratio combining, the CTIR is given by

$$
\bar{\beta}_{i}(t)=\sum_{k \in K_{i}} \frac{\bar{g}_{i k}(t) \bar{p}_{i}(t)}{\bar{I}_{k}^{\mathrm{tot}}(t)}
$$

Conversely, when connected to different cell sites as in soft handover, selection combining is used and the signal with the best quality measure is chosen.

\section{Relative Load and Uplink Admission Control}

In this section, we will derive a practical estimate of the relative load from literature, to form a tractable admission control algorithm. For notational ease, the time index $t$ will be suppressed throughout the section.

\section{A. Relative Load}

The maximal load of a base station in a sparsely planned second generation TDMA/FDMA system is solely determined by the number of frequency channels (times the number of time slots) at that base station. In densely planned systems such as a WCDMA system or a GSM system with all available frequency channels at every base station, the maximal load is determined by the propagation situation between mobile and base stations. Therefore, the capacity of the system is to its nature soft and varying but slowly with time.

A number of approximations will be adopted to obtain a relative load estimate based on available measurements. Assume that the CTIR expression for softer handover in 3 is approximately true also for soft handover. Note that the assumption is not that maximum ratio combining is always used but rather that it constitutes an approximate expression, which is more true when the active set is dominated by one connection. This yields

$$
\bar{\beta}_{i}=\bar{p}_{i} \sum_{k \in K_{i}} \frac{\bar{g}_{i k}}{\overline{\bar{I}}_{k}^{\mathrm{tot}}} \Longleftrightarrow \bar{p}_{i}=\frac{\bar{\beta}_{i}}{\sum_{k \in K_{i}} \overline{\bar{g}}_{k} \overline{\bar{I}}_{k}^{\mathrm{ot}}}, \forall i
$$

Combining Equations (2) and (4), assume perfect inner-loop power control $\left(\bar{\beta}_{i}=\bar{\beta}_{i}^{t}\right)$ results in

$$
\bar{I}_{j}^{\mathrm{tot}}=\bar{v}_{j}+\sum_{i=1}^{M} \bar{g}_{i j} \bar{p}_{i}=\bar{v}_{j}+\sum_{i=1}^{M} \frac{\bar{g}_{i j} \bar{\beta}_{i}^{t}}{\sum_{k \in K_{i}} \overline{\bar{g}}_{i k}^{\mathrm{ot}}}
$$

Furthermore, we elaborate with the temporary approximation that the total interference powers at the different base stations are equal, $\bar{I}_{k}^{\text {tot }}=\bar{I}_{j}^{\text {tot }}$. Solve for $\bar{I}_{j}^{\text {tot }}$, yields

$$
\bar{I}_{j}^{\mathrm{tot}}=\frac{\bar{v}_{j}}{1-\sum_{i=1}^{M} \frac{\bar{\beta}_{i}^{t} \bar{g}_{i j}}{\sum_{k \in K_{i}} \bar{g}_{i k}}}
$$

This expression relates to the discussion in [1], [2], [19], [21], where the total interference at base station $j$ is expressed as

$$
\bar{I}_{j}^{\mathrm{tot}}=\frac{\bar{v}_{j}}{1-\bar{L}_{j}},
$$

In those references, the relative or fractional load $\bar{L}_{j}$ is primarily seen as an alternative representation of the ratio $\bar{I}_{j}^{\text {tot }} / \bar{v}_{j}$, 
referred to as the noise raise.

$$
\frac{\bar{I}_{j}^{\text {tot }}}{\bar{v}_{j}}=\frac{1}{1-\bar{L}_{j}} \Longleftrightarrow \bar{L}_{j}=1-\frac{1}{\bar{I}_{j}^{\text {tot }} / \bar{v}_{j}} .
$$

For example, the objective in [1] is a noise raise of $4 \mathrm{~dB}$, which corresponds to $\bar{L}_{j} \approx 0.6$. An intuitive relative load interpretation is that $\bar{L}_{j}=0$, which means that the interference is only thermal noise, corresponds to the situation when the cell is not loaded. Conversely, $\bar{L}_{j}=1$ is synonymous to infinite interference power and thus an upper load limit.

Hence, we conclude from Equation (6) that it therefore is relevant to introduce the following relative load estimate $\hat{\bar{L}}_{j}$, which is based on available measurements

$$
\hat{\bar{L}}_{j}=\sum_{i=1}^{M} \frac{\bar{\beta}_{i}^{t} \bar{g}_{i j}}{\sum_{k \in K_{i}} \bar{g}_{i k}},
$$

\section{B. An Uplink Admission Control Strategy}

The relative load estimate in (9) can be used to determine whether there is room for yet another mobile station in the system. It is therefore of value for admission control. However, power gain measurements are needed. They can be made available in two different ways:

M1: The mobile stations are requested to periodically (but not necessarily synchronously) report pilot power measurements from the e.g., five strongest base stations at a rate of for example $0.5 \mathrm{~Hz}$.

M2: For handover purposes, the mobile typically reports similar measurements in an event-driven fashion. It measures the pilot powers from the neighboring cells and reports up to the six strongest power gains at handover events [22].

In both cases, the channel is assumed reciprocal, i.e., the uplink power gain is assumed approximately equal (at least with respect to path gain and the shadow fading) to the corresponding downlink power gain. Furthermore, these different strategies only provide data from a limited set of mobile-base power gains. The remainder, however, are considered small and set equal to zero.

When a mobile station requests admission at base station $j$ for a service corresponding to a target SIR $\bar{\gamma}_{i}^{t}$, the resulting load can thus be predicted (assuming that the mobile can provide power gain measurements in the admission request procedure.). The following algorithm summarizes the admission control strategy:

\section{Algorithm: Uplink Admission Control Using a Relative Load Estimate}

Input: List of neighboring cells $\mathcal{N}_{j}$, Power gain measurements from the mobiles including the mobile requesting admission in cell $j$ (gains to distant base stations not included in the measurement report from the mobile are considered zero), CTIR targets $\bar{\beta}_{i}^{t}$.

i) Predict the new load in cell $j$ and the set of neighboring cells $\mathcal{N}_{j}$ to cell $j$ using $\hat{\bar{L}}_{j}=\sum_{i} \frac{\bar{g}_{i j} \bar{\beta}_{i}^{t}}{\sum_{k \in K_{i}} \bar{g}_{i k}}$ ii) Admit the user only if $\hat{\bar{L}}_{j}$ and $\hat{\bar{L}}_{n}, n \in \mathcal{N}_{j}$ all are below $\delta_{\text {SC}}$.

\section{Simulations}

The aim with the simulations is to validate the relative load estimate and to illustrate the behavior when it is used in admission control. A hard capacity algorithm is used as a reference. Both single- and multi-service scenarios are simulated.

\section{A. Single Service}

Consider a WCDMA-type system with the only service $192 \operatorname{kbps}\left(\gamma^{t}=-8.5 \mathrm{~dB}\right)$ streaming data (chosen to create a considerable load while limiting the number of users to ease the computational burden). The simulated scenario describes a suburban situation with uniform traffic, 21 base stations, intermediate velocities $(50 \mathrm{~km} / \mathrm{h})$, path loss exponent $\alpha=3.5$, shadow fading $\left(d_{0}=30 \mathrm{~m}, \sigma=6 \mathrm{~dB}\right)$, fast fading and RAKE receivers. Measurements of power gains are subject to additive errors with $3 \mathrm{~dB}$ variance. To maintain a high load situation, there are always new users waiting for admission in every cell. The hard capacity algorithm admits the user only if the number of links (in a soft handover, the mobile has a $1 / N$ link to each of the $N$ connected base stations) is less than $\delta_{\mathrm{HC}}$.

To examine the validity of the approximations that led to the load estimate in (9), the corresponding noise raise is computed using (8) and compared to the noise raise in the simulation:

$$
\left[\frac{\bar{I}_{j}^{\mathrm{tot}}}{\bar{v}_{j}}\right]_{\mathrm{dB}}-\left[\frac{1}{1-\hat{\bar{L}}_{r}}\right]_{\mathrm{dB}}
$$

The result in different load situations and for the tractable parameter interval $\delta_{S C} \in[0,0.7]$ is described by Figure 1 . We note that the estimator is biased and that the bias increases with the load. The bias is in fact better with M2 measurements, and the accuracy is acceptable up to $\delta_{S C} \approx 0.5$. By

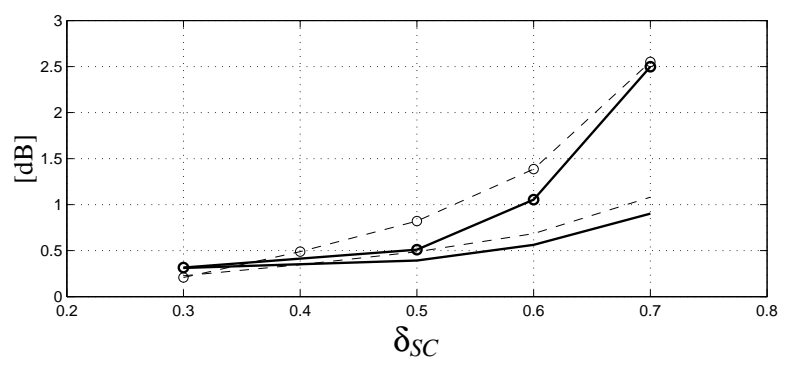

Fig. 1. Mean ('o') and standard deviation of the noise raise estimation error in (10) using measurements accoring to M1 (dashed line) and M2 (thick line) in different load situations.

varying the design parameters $\delta_{S C}$ and $\delta_{H C}$, the relation between how many users that are accommodated and the resulting worst case noise raise (represented by the 90 percentile) can be formed. As seen in Figure 2, admission control utilizing the relative load estimate is tractable. Moreover, we note that it does not seem to be motivated to force the system to report additional measurements (M1). The event-driven measurements (M2) provide similar performance. Users close to 
a base station will report very seldom, but on the other hand, they are relatively cheaply accommodated compared to users on the cell borders. The outer, more costly, users in the cells will on the other hand report relatively frequently. Hence, the measurements are updated more frequently where needed. It is interesting to note that hard capacity yields almost the same performance as soft capacity with uniform traffic. The parameters $\delta_{S C}=0.5$ and $\delta_{H C}=3$ correspond to a 90-percentile noise raise of $4 \mathrm{~dB}$ in Figure 2 .

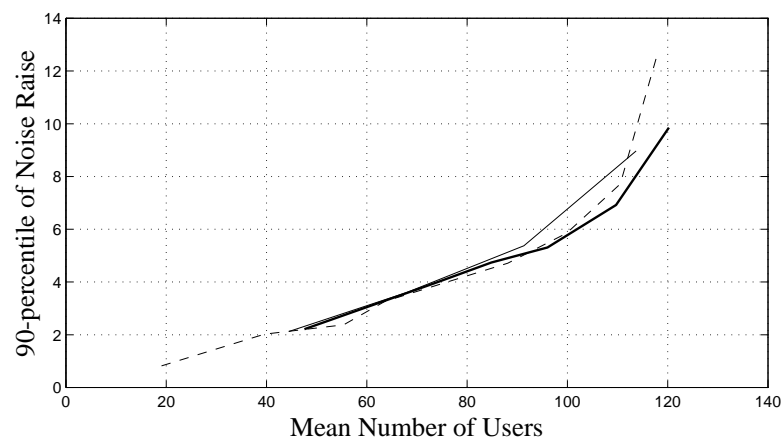

Fig. 2. The relation between accommodated $192 \mathrm{kbps}$ users and resulting noise raise using the three algorithms: soft capacity, M1 (dashed line), soft capacity, M2 (thick line) and hard capacity (thin line).

Basically the same conclusion holds when studying $64 \mathrm{kbps}$ $\left(\gamma^{t}=-17.5 \mathrm{~dB}\right)$ users. Figure 3 shows that soft capacity operates with slightly less noise raise with the same mean number of users. The parameters $\delta_{S C}=0.5$ and $\delta_{H C}=20$ corresponds to a noise raise of $4 \mathrm{~dB}$ (90-percentile). This emphasizes one

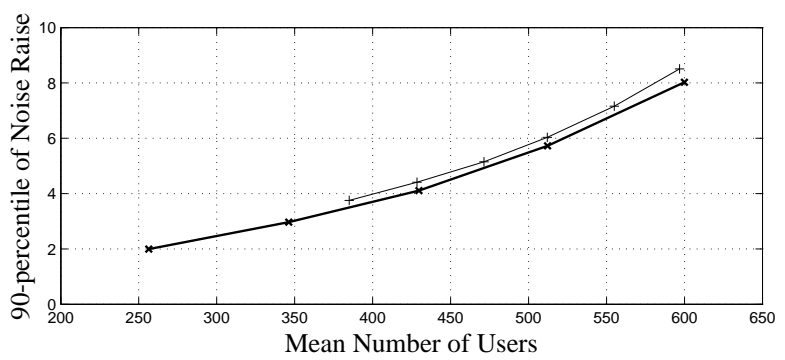

Fig. 3. The relation between accommodated $64 \mathrm{kbps}$ users and resulting noise raise using soft capacity $\mathbf{M} 2$ (thick line) with $\delta_{S C} \in\{0.3,0.4,0.5,0.6,0.7\}$ and hard capacity (thin line) with $\delta_{H C} \in\{18,20,22,24,25,26,28\}$.

of the main benefits with soft capacity - the parameter setting is universal and independent of the services in question. Figure 4 a further indicates that the same parameter value can successfully be used with both services. However, a small deviation can be noted and the low data rate users tend to result in a higher noise raise. This is due to the trouble of filling up the system tightly to the load limit when only high data rate users are available. In real systems, the traffic mix reduces such deviations. Moreover, Figure $4 \mathrm{p}$ illustrates the robustness to different radio enviroments in terms of the path loss exponent. The soft capacity parameter setting is unaffected, a)

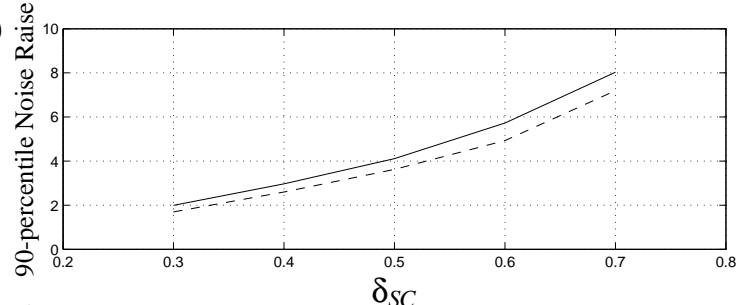

b)

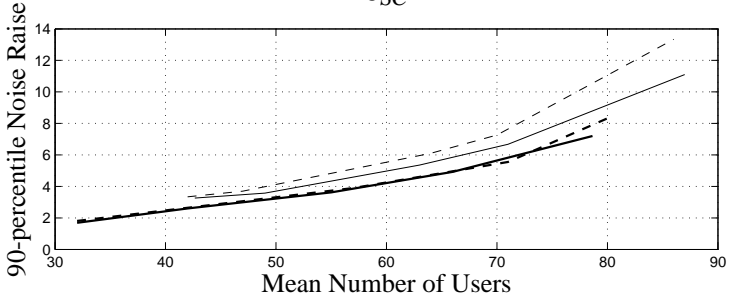

Fig. 4. a) Relations between the design parameter $\delta_{S C}$ and resulting 90-percentile of noise raise for the two services $192 \mathrm{kbps}$ (dashed) and $64 \mathrm{kbps}$ (solid). b) Resulting noise raise (192 kbps users) with soft capacity (thick lines) and hard capacity (thin lines) for the path loss exponents $\alpha=3.5$ (solid) and $\alpha=3.2$ (dashed).

while the hard capacity parameter show a slight dependency.

\section{B. Multi-services}

Since the service requirements of the users are described by the corresponding target CTIR:s, $\bar{\beta}_{i}^{t}$, multi-services are naturally considered. We will use two services: the high data rate user from the previous section, now moving at $3 \mathrm{~km} / \mathrm{h}$, and $12.2 \mathrm{kbps}$ voice users $\left(\gamma^{t}=-22 \mathrm{~dB}\right)$ moving at $50 \mathrm{~km} / \mathrm{h}$ on average. Since the high data-rate users are more costly for the system, we assume that they will be admitted with limited coverage. This is truly the case as seen in Figure 5, which shows the histogram of the power gains for users admitted after an initial phase where the system is filled up. Figure 6

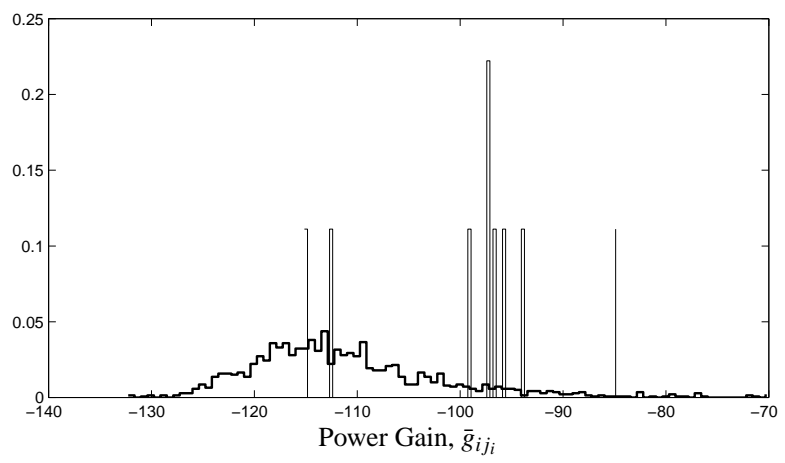

Fig. 5. Histograms of power gains for the services: $12.2 \mathrm{kbps}$ (thick line) and $192 \mathrm{kbps}$ (thin line).

provides more information from two-service simulations (with $\left.\delta_{S C}=0.5\right)$. The algorithm manages to keep the load in terms of worst case noise raise at a steady level (Figure 6a). It is also interesting to note (Figure 6p,c) that the voice users eagerly fill up the system when a high data-rate user disconnects. The 
total number of voice and data users are found in Figure $6 \mathrm{~d}$. One way of increasing the fraction of data users could be to use different thresholds $\delta_{S C}$ for data and for voice, and thereby reserve capacity to some services.

a)

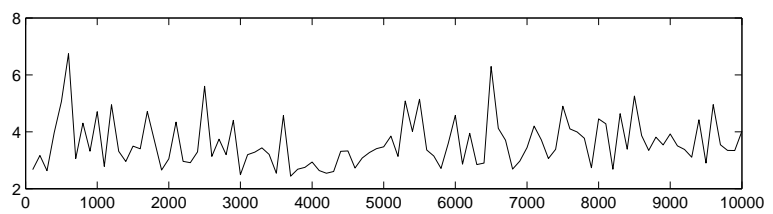

b)

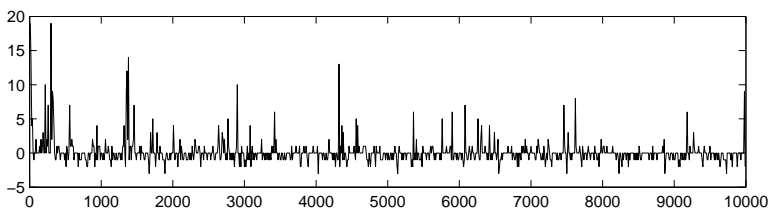

c)

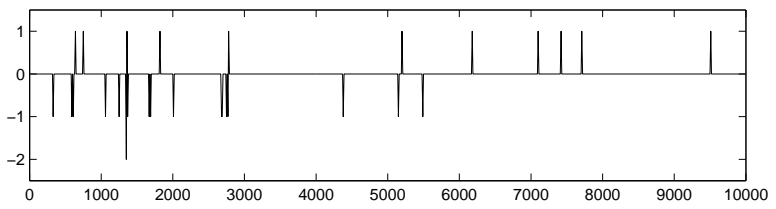

d)

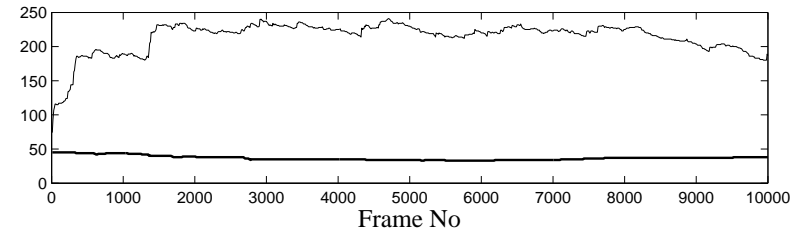

Fig. 6. Simulation results using soft capacity, $\mathbf{M 2}$ and $\delta_{S C}=0.5$. a) Max noise raise (in $\mathrm{dB}$ ) over 100 frames and all base stations. b) Change of voice users over 10 frames. c) Same as b), but for data users. d) Total number of voice and data (thick line) users over time.

\section{Conclusions}

The proposed uplink admission control algorithm utilizes measurement readily available in the system. It is therefore an interesting alternative to algorithms based on interference power measurements, whose accuray is known to be crude and time varying. Another benefit with relative load based admission control is the universal parameter setting. Hard capacity algorithms may provide almost the same performance, but the parameter setting is difficult and service dependent.

The proposed algorithm is tested both with enforced periodical measurements from the mobile stations, and with eventdriven handover measurements readily available, and the performance is roughly the same. It can therefore be employed without significantly increasing the reporting load of the system. Multi-services are naturally handled, and a direct consequence is limited coverage for higher data-rate services compared to lower data-rate. The admitted traffic mix can, however, be altered by admitting higher data-rate users more easily.

\section{DisClaimer}

The simulations are rudimentary and the presented performance in absolute numbers have little relations to real systems. Also the service definitions and interpretations are fictious and serve an illustrative purpose.

\section{REFERENCES}

[1] H. Holma and J. Laakso. Uplink admission control and soft capacity with MUD in CDMA. In Proc. IEEE Vehicular Technology Conference, Amsterdam, the Netherlands, Sep 1999.

[2] C. Y. Huang and Yates R. D. Call admission in power controlled CDMA systems. In Proc. IEEE Vehicular Technology Conference, Atlanta, GA, USA, May 1996.

[3] Y. Ishikawa and N. Umeda. Capacity design and performance of call admission control in cellular CDMA systems. IEEE Journal on Selected Areas in Communications, 15(8), 1997.

[4] Z. Lei, D. Goodman, and N. Mandayam. Location-dependent other-cell interference and its effect on the uplink capacity of a cellular CDMA system. In Proc. IEEE Vehicular Technology Conference, Houston, TX, USA, May 1999.

[5] J. Kuri and P. Mermelstein. Cell admission on the uplink of a CDMA system based on total received power. In Proc. IEEE International Conference on Communications, Vancouver, BC, Canada, June 1999.

[6] N. Dimitriou, G. Sfikas, and R. Tafazolli. Call admission policies for UMTS. In Proc. IEEE Vehicular Technology Conference, Tokyo, Japan, May 2000.

[7] J. Outes, L. Nielsen, K. Pedersen, and P. Mogensen. Multi-cell admission control for UMTS. In Proc. IEEE Vehicular Technology Conference, Rhodes, Greece, May 2001.

[8] B. Timus and J. Pettersson. Uplink admission control for conversational services using information from many cells. In Proc. IEEE Vehicular Technology Conference, Rhodes, Greece, May 2001.

[9] J. Choi and S. Bhak. Multiclass call admission control in QoS-sensitive CDMA networks. In Proc. IEEE International Conference on Communications, Helsinki, Finland, June 2001.

[10] Z. Liu and M. El Zarki. SIR-based call admission control for DS-CDMA cellular systems. IEEE Journal on Selected Areas in Communications, 12(4), 1994

[11] I. M. Kim, B. C. Shin, and D. J. Lee. SIR-based call admission control by intercell interference prediction for DS-CDMA systems. IEEE Communications Letters, 4(1), 2000.

[12] S. Sun and W.A. Krzymien. Call admission policies and capacity analysis of a multi-service CDMA personal communication system with continuous and discontinuous transmission. In Proc. IEEE Vehicular Technology Conference, Ottawa, Canada, May 1998.

[13] T. K. Liu and J. A. Silvester. Joint admission/congestion control for wireless CDMA systems supporting integrated services. IEEE Journal on Selected Areas in Communications, 16(6), 1998.

[14] C. Comaniciu, N. Mandayam, D. Famolari, and P. Agrawal. Admission and flow control for multimedia CDMA. In Proc. IEEE International Conference on Multimedia and Expo, New York, NY, USA, July 2000.

[15] N. Dimitriou, R. Tafazolli, and G. Sfikas. Quality of service for multimedia CDMA. IEEE Communications Magazine, 38(7), 2000.

[16] S. V. Hanly. Congestion measures in DS-CDMA. IEEE Transactions on Communications, 47(3), 1999.

[17] F. Gunnarsson. Power Control in Cellular Radio System: Analysis, Design and Estimation $\mathrm{PhD}$ thesis, Linköpings universitet, Linköping, Sweden, April 2000.

[18] F. Gunnarsson and F. Gustafsson. Power control with time delay compensation. In Proc. IEEE Vehicular Technology Conference, Boston, MA, USA, Sep 2000.

[19] N. Wiberg and A. Gioia. Uplink packet access control in WCDMA. In Proc. IEEE Vehicular Technology Conference, Tokyo, Japan, May 2000.

[20] E. Geijer Lundin. Uplink admission control based on estimated interference in WCDMA systems. Master Thesis LiTH-ISY-EX-3123, Dept. of Electrical Engineering, Linköpings universitet, Sweden, 2001.

[21] J. Shapira. Microcell engineering in CDMA cellular networks. IEEE Transactions on Vehicular Technology, 43(4), 1994.

[22] 3GPP Technical Specification Group Radio Access Network. Requirements for support of radio resource management. Technical report $3 \mathrm{G}$ TS 25.133, 2000 